\title{
Entropic considerations on the Universe and Universe-black hole systems
}

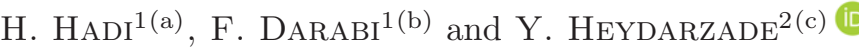 \\ 1 Department of Physics, Azarbaijan Shahid Madani University - 53714-161 Tabriz, Iran \\ 2 Department of Mathematics, Faculty of Sciences, Bilkent University - 06800 Ankara, Turkey
}

received 13 July 2020; accepted in final form 21 August 2020

published online 22 September 2020

PACS 98.80.Jk - Mathematical and relativistic aspects of cosmology

PACS 95.30.Sf - Relativity and gravitation

\begin{abstract}
We study the entropic considerations on the Universe system and the Universe-black hole system, filled by cosmological constant or exotic quintessence-like and phantom-like fields having negative pressure, using their relevant entropic bounds. It turns out that for both systems these considerations single out the cosmological constant, among the negative pressure candidate fields, as the viable cosmological field.
\end{abstract}

Copyright (C) 2020 EPLA

Introduction. - The cosmological constant, as a constant energy density in space and time, is the simplest candidate for dark energy. Actually, the $\Lambda$-Cold Dark Matter $(\Lambda C D M)$ model is consistent with the current observations, however, this model suffers from the cosmological constant problem [1] and coincidence problem [2]. These problems have been tackled by introducing some models of dark energy, the most relevant of which are quintessence and phantom fields. The quintessence, with the equation of state $-1<\omega_{q}<-\frac{1}{3}$, as a canonical scalar field with a particular potential can describe the late-time cosmic acceleration [3]. What is considerable about the quintessence field is that its equation of state dynamically changes with time. Quintessence field has a prominent role in cosmological dynamics in the presence of matter and radiation [4-7]. The other candidate for dark energy is the phantom field, with the equation of state $\omega_{p}<-1$, which can also describe the current cosmological dynamics [8-10]. The equation-of-state parameter for the phantom field, when approaches a constant value, results in a big-rip singularity which itself is a new problem. Although all these three models of dark energy are capable of predicting an accelerating cosmological dynamics, their imperfection for a perfect description of current cosmic dynamics motivates one to revisit these dark energy models from a non-cosmic point of view. Here, it

\footnotetext{
(a) E-mail: hamedhadi1388@gmail.com

(b) E-mail: f.darabi@azaruniv.ac.ir

(c) E-mail: yheydarzade@bilkent.edu.tr
}

is worth mentioning that there are also other attempts suggesting an extension of Einstein's general relativity in order to explain the current accelerating expansion of the Universe [11]. What is known as an extended theory of general relativity is a semi-classical theory with a modification in the Einstein-Hilbert action. For instance, this modification is done by considering higher-order curvature invariant terms or by adding terms where a scalar field is coupled non-minimally to geometry $[12,13]$. In this framework, $f(R)$ gravity [14] is one of the most popular models addressing both dark energy and dark matter problems. In [11], the possibility of testing the framework of extended theory of gravity using gravitational waves is anticipated. This may be considered as an important step forward in the framework of extended theories of gravity because of the recent progresses in the gravitational wave astronomy through the famous LIGO detections.

Another point of view is the powerful thermodynamical approach and the entropic considerations for the study of dark energy models. It is well known that in principle the equations of motion can predict the future of time-reversible physical systems, however, in reality, timereversibility is not seen for thermodynamical systems due to the entropic consideration. Two relevant examples of such thermodynamical systems in the present study are the Padmanabhan's emergent paradigm and Universeblack hole system surrounded by cosmological fields. In explicit words, we explore the cosmological fields by entropic considerations and investigate which cosmological field as a candidate for dark energy model can be preferred 
by such entropic considerations in the study of the Universe system and Universe-black hole system.

The idea that gravity behaves as an emergent phenomenon is referred to the proposal made by Sakharov in 1967 [15]. In this proposal, named the induced gravity, the spacetime background emerges as a mean-field approximation of some underlying microscopic degrees of freedom, very similar to hydrodynamics or continuum elasticity theory from molecular physics [16]. Current research works on the relation between gravity and thermodynamics support this point of view [17], where the major attention is focused on how the gravitational field equations can be derived from the thermodynamical point of view.

In a pioneering work of Jacobson, the Einstein field equations were obtained, using the equivalence principle and Clausius relation $\mathrm{d} Q=T \mathrm{~d} S$ where $Q, T$ and $S$ are the heat, temperature and entropy, respectively [18]. The key point in this work was to demand that the Clausius relation should hold for all local Rindler causal horizons with $Q$ and $T$ interpreted as the energy flux and Unruh temperature, as seen by an accelerated observer located inside the horizon. In this thermodynamic approach, the Einstein field equations appear as the equations of state of spacetime. The Clausius relation also arises when one treats the gravitational field equations as an entropy balance law across a null surface, i.e., $S_{m}=S_{\text {grav }}$ [19].

In another work by Padmanabhan, as a new approach to show a relation between gravity and thermodynamics, the gravity was shown not to be a fundamental interaction [20]. In this approach, Newton's law of gravitation was derived by combining the equipartition law of energy for the horizon degrees of freedom with the thermodynamical relation $S=\frac{E}{2 T}$ where $S, T$ and $E$ are entropy, horizon temperature and active gravitational mass, respectively [21]. It was also argued that the current accelerated expansion of the Universe may be derived from the discrepancy between the surface and bulk degrees of freedom through the relation $\Delta V / \Delta t=N_{\text {sur }}-N_{\text {bulk }}$, where $N_{\text {bulk }}$ and $N_{\text {sur }}$ are the degrees of freedom related to matter-energy content inside the bulk and surface area, respectively [22]. These studies magnify the importance of thermodynamic approach to gravity as well as the corresponding thermodynamical quantities, even for cosmological systems. The entropy and its bounds play the key roles in these kinds of studies.

The application of Bekenstein's bound to sufficiently small regions of the Universe can be found in [23-29]. Moreover, Fischler and Susskind have proposed a bound [24] which can formulate the holographic principle [30-32]. They have carefully exposed the difficulties that arise when such bounds are pushed beyond their range of validity $[24,25,28]$. On the other hand, Bousso has proposed the D-bound for systems with cosmological horizon, like asymptotically non-flat Schwarzschild-de Sitter black hole [33]. Of course, one can look for the D-bound for other solutions which are not asymptotically flat and include a cosmological apparent horizon. A general proposal was then suggested by Bousso [34] for entropy bound, the so-called covariant entropy bound, which has been shown to comply with Bekenstein's entropy bound and the Fischler and Susskind bound.

In this paper, we will consider the system "Universe" and the system "Universe-black hole" filled by some exotic fields, and investigate those fields that can be preferred by imposing the relevant entropy bounds on these systems. First, for the system "Universe", we will apply the covariant entropy bound on the light-like cosmological horizon and the entropy bound arising from the Padmanabhan's emergent paradigm. These two entropy bounds should be identified on the light-like cosmological horizon. The identification of the D-bound and the Bekenstein bound, in the framework of the surrounded Universe-black hole system, can be considered as a suitable criterion by which one can single out the exotic cosmological fields in agreement with this criterion and rule out the other cosmological fields in disagreement with this criterion. In doing so, for the system "Universe-black hole" we will apply the D-bound and the Bekenstein entropy bound on the black hole. These two entropy bounds should also be identified on the black hole. From the mentioned identifications, we will obtain the preferred exotic fields as the viable cosmological fields. In the next section, we shall find an entropy bound which is resulted by means of Padmanabhan's emergent paradigm and we attempt to identify the maximum entropy bound coming from the covariant entropy conjecture, on the one hand, and the entropy of Padmanabhan's emergent paradigm on the other hand for the Universe system. In the third section, the D-bound and Bekenstein bound are studied for the exotic quintessence-like and phantom-like fields, respectively, and their identifications are investigated for the Universe-black hole system. Finally, in the fourth section, we give our concluding remarks.

Entropy bounds for the Universe system. - The relevant entropy bounds which are of particular importance in the present study for the Universe system are "entropy bound of Padmanabhan's emergent Universe" and Bousso's "covariant entropy bound", as is described in the following argument. From observational evidences we know that the universe is almost flat, namely $k=0$, for which the Hubble horizon in Padmanabhan's paradigm becomes exactly the same as the apparent horizon for a flat Universe. The covariant entropy bound is introduced based on the "light-sheets" (null surfaces) [34], and, on the other hand, the Hubble horizon in the Padmanabhan's emergent paradigm becomes a "null apparent horizon" specifically for a flat Universe. Therefore, the Hubble horizon in the Padmanabhan's emergent paradigm for a flat universe can be considered as a system for which one can ascribe the covariant entropy bound. In other words, if $k=0$ is provided, then the Hubble horizon in Padmanabhan's paradigm plays the role of null surface enclosing the 
Universe and the covariant entropy bound becomes applicable to this system. For details of discussion, refer to the cosmological corollary of covariant entropy bound in [34].

Entropy bound for Padmanabhan's emergent flat Universe. In this subsection, we show that how Padmanabhan's emergent paradigm for an expanding flat universe can be written as an "emergent lower entropy bound" for the Universe system.

According to Padmanabhan's proposal, the difference between the surface degrees of freedom and the bulk degrees of freedom in a region of space may result in the accelerated expansion of the Friedmann-RobertsonWalker (FRW) Universe through the relation $\Delta V / \Delta t=$ $N_{\text {sur }}-N_{\text {bulk }}$, where $N_{\text {bulk }}$ and $N_{\text {sur }}$ are referred to the degrees of freedom related to matter and energy content inside the bulk and surface area, respectively [22].

For an expanding Universe, we have the following condition for the Padmanabhan's formula:

$$
\frac{\Delta V}{\Delta t} \geqslant 0
$$

which demands

$$
N_{\text {sur }}-N_{\text {bulk }} \geqslant 0 \text {. }
$$

On the other hand, we know that the relation between surface entropy $S_{\text {sur }}$ and surface degrees of freedom is as follows:

$$
4 S_{\mathrm{sur}}=N_{\mathrm{sur}}
$$

where the entropy of the surface is $\frac{A}{4}, A$ being the area of the surface enclosed by the Hubble horizon $r_{H}$. One can also write the bulk degrees of freedom in terms of its energy $E$ and temperature $T$ as

$$
N_{\text {bulk }}=\frac{2 E}{T}
$$

where the thermodynamic temperature of our cosmological system is $H / 2 \pi$. So, one can rewrite the equations (2), (3) and (4) as follows:

$$
\pi r_{H} E \leqslant S_{\text {sur }}
$$

which can be interpreted as a definition of the "emergent lower entropy bound". The reason why we call (5) as the emergent lower entropy bound is that it is a trivial rewriting of the Friedmann equation in terms of non-standard variables $r_{H}, E$ and $S$, and has no independent content. For example, unlike $S$ in the covariant bound, Padmanabhan's $N_{\text {bulk }}$ is not defined as the von Neumann entropy or the thermodynamic entropy of an actual bulk matter system, rather it is just a suggestive name given to a quantity that is directly defined in terms of quantities like $H, \rho$, and $p$ that appear in the Friedmann equation. So, there is no non-trivial content to the statement that the Friedmann equation can be expressed in terms of such quantities. That is why the relation (5) cannot be considered as a definition of a "lower entropy bound" for the surface entropy $S_{\text {sur }}$, so it merely can be interpreted as a definition of "emergent lower entropy bound" of a cosmological system in the framework of the emergent Universe scenario. Therefore, it is meaningless to compare in general the "covariant upper entropy bound" with " $\pi r_{H} E$ as the emergent lower bound of $S_{\text {sur }}$ ", unless some specific conditions are provided in order for this comparison to become meaningful. In the following section, we shall discuss that there is one specific possibility providing a meaningful comparison which corresponds to a spatially flat universe, i.e., $k=0$.

Covariant entropy bound for the flat Universe. In order to apply the covariant entropy bound on the flat Universe, one should study the relevant energy contents of the flat Universe, namely Misner-Sharp energy and Komar energy, and investigate which of these two is successful in matching the covariant entropy bound with the entropy bound of Padmanabhan's emergent paradigm, for the flat Universe.

Misner-Sharp energy: Let us start with the MisnerSharp energy [35]

$$
E=\int T_{\mu \nu} u^{\mu} u^{\nu} \mathrm{d} V
$$

where $u^{\mu}=\delta_{0}^{\mu}, T_{\mu \nu}$ and $V$ are the energy-momentum source and the volume of the bulk space, respectively. Then, the total Misner-Sharp energy inside the Hubble horizon reads

$$
M\left(r_{H}\right)=\int_{0}^{r_{H}} 4 \pi r^{2} \rho \mathrm{d} r=\frac{4 \pi}{3} r_{H}^{3} \rho,
$$

where $r_{H}$ is the Hubble horizon radius and $M=E$. Moreover, for the apparent horizon we have $r=2 M(r)$ in which for our cosmological case with a flat spatial geometry the apparent and Hubble horizons coincide and consequently this formula takes the form of $r_{H}=2 M\left(r_{H}\right)$. Also, using the Friedmann equations for $k=0$, we have $r_{H}=\sqrt{\frac{3}{8 \pi \rho}}$. Then, using (5) and (7), we obtain

$$
\frac{\pi r_{H}^{2}}{2} \leqslant S_{\text {sur }}
$$

On the other hand, the maximum of Bousso's covariant entropy bound for $k=0$ and the null surface to be defined by $r_{H}$ is given by

$$
S=\frac{A}{4}=\pi r_{H}^{2}
$$

We demand that the inequality (5) to be saturated for $k=0$ and the null surface defined by $r_{H}$ as

$$
S_{\text {sur }}=\pi r_{H} E \text {, }
$$

such that it can be compared with (9) on the null surface defined by $r_{H}$. In doing so, if we put the Misner-Sharp energy $E=\frac{r_{H}}{2}$ in (10), we arrive at the result that the Misner-Sharp energy has no capability for having the equal values of entropy bounds (9) and (10) on the "null surface of cosmological apparent horizon". Therefore, this energy definition fails in matching two entropy bounds. 
Komar energy: In this subsection, we show that Komar energy is capable for removing the above-mentioned inconsistency for the Misner-Sharp energy. To begin with, we consider the Komar energy [36]

$$
E=\int\left(2 T_{\mu \nu}-T g_{\mu \nu}\right) u^{\mu} u^{\nu} \mathrm{d} V
$$

where $T$ is the trace of the energy-momentum source $T_{\mu \nu}$. Then, the total Komar energy in the bulk space enclosed by the surface of the Hubble horizon is given by [22]

$$
E\left(r_{H}\right)=\int_{0}^{r_{H}} 4 \pi r^{2}|\rho+3 p| \mathrm{d} r=\frac{4 \pi}{3} r_{H}^{3} \rho|1+3 \omega|,
$$

where we have considered the barotropic equation of state $p=\omega \rho\left(\right.$ see footnote $\left.^{1}\right)$. Then, from the inequality (5), we obtain

$$
4 \pi r_{H}\left(\frac{4 \pi}{3} r_{H}^{3} \rho\right)|1+3 \omega| \leqslant A=4 S
$$

where the L.H.S. becomes maximum (equality case) at $r_{H}$ as

$$
A=4 S_{\text {sur }}=2 \pi r_{H}\left(2 M\left(r_{H}\right)\right)|1+3 \omega| .
$$

Then, using (14), we obtain

$$
S_{\text {sur }}=\frac{\pi r_{H}^{2}|1+3 \omega|}{2} .
$$

This shows that, unlike the Misner-Sharp energy, the Komar energy has capability for having the equal values of entropy bounds (9) and (15) on the cosmological apparent horizon null surface for two specific values of $\omega$ as $\omega=1 / 3$ and $\omega=-1$ corresponding to radiation and de Sitter universes, respectively.

It is interesting to note that without comparison between the covariant entropy bound and entropy bound which comes from Padmanabhan's emergent paradigm, one cannot reach the correct relation (15) between the entropy of the enclosing surface and the physical quantities within the bulk space. This relation indicates the holographic behaviour of the system in a beautiful way and shows that the holographic principle demands the Komar energy as the correct energy content of the cosmological bulk space.

In the following section, based on what we obtained, we discuss that one may obtain the current accelerating expansion of the Universe as a matching condition of two cosmological entropy bounds resulting from the covariant bound and emergent paradigm.

\footnotetext{
${ }^{1}$ Regarding that both the degrees of freedom and entropy are positive definite quantities, the absolute value in the integrand in (12) is considered for the positivity of the bulk degrees of freedom in eq. (4) as well as for the entropy in eq. (15). In fact, one has to consider the absolute value of energy or follows Padmanabhan [22] by introducing the $\epsilon$ parameter to assure the positivity of the bulk degrees of freedom for both the positive or negative values of $\rho+3 p$, see eq. (8) in $[22]$.
}

Entropy bounds for the Universe-black hole system filled by cosmological fields. - The relevant entropy bounds which are of particular importance in the present study for the Universe-black hole system filled by cosmological fields are the D-bound and Bekenstein bound. This is because both the D-bound and Bekenstein bound are applicable on this system having both event horizon and cosmological apparent horizon. This feature lets us to compare the D-bound with the Bekenstein bound for the Universe-black hole system filled by cosmological fields.

The black hole inside the Universe-black hole system filled by cosmological field is considered as a solution surrounded by the cosmological field. The metric of this solution is given by [37]

$$
\begin{aligned}
\mathrm{d} s^{2}= & -\left(1-\frac{r_{g}}{r}-\left(\frac{r_{s}}{r}\right)^{3 \omega_{s}+1}\right) \mathrm{d} t^{2} \\
& +\frac{\mathrm{d} r^{2}}{\left(1-\frac{r_{g}}{r}-\left(\frac{r_{s}}{r}\right)^{3 \omega_{s}+1}\right)}+r^{2} \mathrm{~d} \Omega^{2} .
\end{aligned}
$$

Note that the metric (16) is an exact and a general solution for the Universe-black hole system in the sense that the equation of state of the surrounding cosmological field $p_{s}=\omega_{s} \rho_{s}$ is generic. Going through [37] which specifically studies the exotic quintessence-like field, one finds that the derivation of the solution is completely general and not specific to the quintessence-like field; it can reduce to its specific limits, like the black hole in the radiation or de Sitter background, by the particular choices of the equation-of-state parameter $\omega_{s}$. The main origin and motivation for the Kiselev solution [37] is the current acceleration of the Universe. This implies that such a solution is important from a cosmological point of view, like the Schwarzschild-de Sitter solution, in the sense that it describes a black hole embedded in a cosmological field governing the current accelerating expansion phase of the Universe. This fact motivated us to investigate this solution as a model for the Universe-black hole system. In the Kiselev solution, each arbitrary choice of $\omega_{s}$ represents an arbitrary cosmological background field, and so the solution (16) describes a black hole embedded in that cosmological background field. For example:

- The cases $\omega_{s}=0$ and $\omega_{s}=1 / 3$ represent the dust and radiation backgrounds, see, for example, [38]. These particular limits are also mentioned in [37] at the beginning of section 3 , and by following its derivation one can recognize the generality of this solution.

- By setting $\omega_{s}=-1$, the general solution in [37] reduces to the Schwarzschild-de Sitter solution, the entropy D-bound of which is obtained in [33].

- The case $-1<\omega<-1 / 3$ represents the background exotic quintessence-like field with a negative pressure which is a candidate for dark energy as the source for the current accelerating expansion of the Universe [39]. It is worth mentioning that we name 
this field "quintessence-like" since it has no dynamics, and its energy-momentum source is not a perfect fluid.

- The range $\omega<-1$ corresponds to exotic phantomlike fields with super-negative pressures as other candidates for the dark energy [40]. The final fate of a Universe dominated by a dynamical phantom field is Big Rip [41]. The same argument on the name of quintessence-like field applies for this case.

D-bound and Bekenstein bound for the Universe-black hole system filled by exotic quintessence-like field. In the case of a neutral black hole surrounded by quintessence with $w_{q}=-2 / 3[37]$ the metric (16) becomes

$$
\begin{aligned}
\mathrm{d} s^{2}= & -\left(1-\frac{r_{g}}{r}-\left(\frac{r_{q}}{r}\right)^{-1}\right) \mathrm{d} t^{2} \\
& +\frac{\mathrm{d} r^{2}}{\left(1-\frac{r_{g}}{r}-\left(\frac{r_{q}}{r}\right)^{-1}\right)}+r^{2} \mathrm{~d} \Omega^{2} .
\end{aligned}
$$

The inner and outer horizons are obtained by the equation $g^{r r}=0$ as follows:

$$
\begin{aligned}
r_{\mathrm{in}} & =\frac{1}{2}\left(r_{q}-\sqrt{r_{q}^{2}-4 r_{q} r_{g}}\right), \\
r_{\mathrm{out}} & =\frac{1}{2}\left(r_{q}+\sqrt{r_{q}^{2}-4 r_{q} r_{g}}\right),
\end{aligned}
$$

where $r_{q}>4 r_{g}$ and $r_{g}<r_{\text {in }}<r_{\text {out }}<r_{q}$. For constructing the D-bound [33] we take the following procedure. The Dbound is derived by assuming a matter system inside the apparent cosmological horizon of an observer inside a Universe, with a future de Sitter asymptotic space, which is then converted into an empty pure de Sitter space through a thermodynamical process by which the matter system is pushed outward the cosmological horizon. The total entropy of the asymptotic de Sitter space, including the matter system, as the initial thermodynamical system is given by

$$
S=S_{m}+\frac{A_{c}}{4}
$$

where $S_{m}$ is the entropy of the initial matter inside the cosmological horizon and $A_{c} / 4$ is the Bekenstein-Hawking entropy of the apparent cosmological horizon, enclosing the matter system, with surface area $A_{c}$. The final entropy of the system, after matter evacuation, will be $S_{0}=A_{0} / 4$ in which $A_{0}$ is the area of cosmological horizon of the pure de Sitter space. Considering the generalized second law of thermodynamics $S \leq S_{0}$, we obtain [33]

$$
S_{m} \leqslant \frac{A_{0}}{4}-\frac{A_{c}}{4}
$$

which is the so-called D-bound on the matter system.

In the case of Universe-black hole system filled by exotic quintessence-like field, $A_{0}$ and $A_{c}$ are the area of the horizons enclosing the quintessence-like field and the quintessence-like plus matter fields, respectively. Using $r_{0}=\left.r_{\text {out }}\right|_{m=0}=r_{q}$ and $r_{c}=r_{\text {out }}$, the D-bound becomes

$$
S_{m} \leqslant \pi\left(\frac{r_{q}^{2}}{2}+r_{q} r_{g}-\frac{r_{q}}{2} \sqrt{r_{q}^{2}-4 r_{q} r_{g}}\right) .
$$

For $r_{q} \gg r_{g}$, the D-bound leads to

$$
S_{m} \leqslant 2 \pi r_{q} r_{g}
$$

On the other hand, the Bekenstein bound is given by [33]

$$
S_{m} \leqslant \pi r_{\mathrm{in}} R
$$

where $r_{\text {in }}$ is the gravitational radius of the system and $R=$ $r_{\text {out }}$ is the geometric radius of the system. For $r_{q} \gg r_{g}$ the Bekenstein bound becomes

$$
S_{m} \leqslant \pi r_{q} r_{g}
$$

Therefore, it turns out that in this case the Bekenstein bound and the D-bound are not the same for the dilute system. Indeed, the Bekenstein bound is tighter than the D-bound.

D-bound and Bekenstein bound for the Universe-black hole system filled by exotic phantom-like field. In the case of a neutral black hole surrounded by exotic phantomlike field with $w_{p}=-4 / 3$, the metric (16) reads

$$
\begin{aligned}
\mathrm{d} s^{2}= & -\left(1-\frac{r_{g}}{r}-\left(\frac{r_{p}}{r}\right)^{-3}\right) \mathrm{d} t^{2} \\
& +\frac{\mathrm{d} r^{2}}{\left(1-\frac{r_{g}}{r}-\left(\frac{r_{p}}{r}\right)^{-3}\right)}+r^{2} \mathrm{~d} \Omega^{2} .
\end{aligned}
$$

Because of $g^{r r}=0$ the inner and outer horizons for $r_{p} \gg$ $r_{g}$ are as follows:

$$
\begin{aligned}
r_{\text {in }} & =r_{g}+r_{g}^{4} r_{p}^{-3}+O\left(r_{p}^{-6}\right), \\
r_{\text {out }} & =r_{p}-\frac{r_{g}}{3}-\frac{2 r_{g}}{9 r_{p}}-\frac{20}{81} r_{g}^{3} r_{p}^{-2}-\frac{1}{3} r_{g}^{4} r_{p}^{-3}+O\left(r_{p}^{-4}\right) .
\end{aligned}
$$

To derive the D-bound for this system we use the same equation (21). In this case $A_{0}$ and $A_{c}$ are the areas of the horizons enclosing the exotic phantom-like field and the phantom-like plus matter fields, respectively. For $r_{p} \gg r_{g}$, using $r_{0}=\left.r_{\text {out }}\right|_{m=0}=r_{p}$ and $r_{c}=r_{\text {out }}$, the D-bound is obtained as

$$
S_{m} \leqslant \frac{2}{3} \pi r_{g} r_{p}
$$

The Bekenstein bound (24) for this case is given by

$$
S_{m} \leqslant \pi r_{g} r_{p}
$$

For this case, the D-bound bound is tighter than the Bekenstein bound. It turns out that, as for the exotic quintessence-like field, for the exotic phantom-like field also the Bekenstein bound and the D-bound are not the 
same for dilute systems. Then, regarding the gravitational nature of both the quintessence-like and phantomlike fields in which both of them are violating the strong energy condition but they lead to the looser and tighter D-bounds relative to Bekenstein bound, a question rises up here. Is there any particular field violating the strong energy condition but providing the same result for both these entropy bounds? In other words, what is the matching condition for these two bounds? It is shown by Bousso that for the case of a cosmological constant, both these entropy bounds coincide [33]. Then, regarding our obtained results for the exotic quintessence-like and phantom-like fields along with [33], it is seen that the D-bound and Bekenstein bound coincide for the Universe-black hole system only for the cosmological constant. These findings prove that although all the quintessence-like, cosmological constant and phantom-like fields are motivated for deriving the current accelerating expansion of the Universe, the cosmological constant is the only viable cosmological field from the entropic point of view.

Discussion and concluding remarks. - By applying Bousso's covariant entropy conjecture for the cosmological spatial region, on the one hand, and the entropy bound which comes from the Padmanabhan's emergent paradigm, on the other hand, we have shown that these two entropy bounds are in agreement just for the flat $(k=0)$ FRW Universe and are equal to the maximal entropy on the "null surface" defined by the Hubble horizon $r_{H}$, provided that

- inside of the apparent horizon they be filled by the radiation, namely $\omega=\frac{1}{3}$,

or

- inside of the apparent horizon they be a pure de Sitter space subject to the cosmological constant, namely $\omega=-1$.

In other words, the maximal entropy inside the apparent horizon of the flat FRW Universe occurs when it is filled completely just by the radiation field or cosmological constant. However, considering the fact that the $\omega=\frac{1}{3}$ case cannot describe the accelerating behavior of the Universe, in the context of Padmanabhan's emergent paradigm, we can leave this case and just keep the $\omega=-1$ case. We arrive at the conclusion that the cosmological fields with $\omega \neq-1$, such as phantom-like and quintessence-like ones, are ruled out because of the non-compatibility of the covariant entropy bound and the entropy bound coming from Padmanabhan's emergent paradigm².

The same result is obtained for the Universe-black hole system filled by the cosmological fields as follows. We know that the D-bound and Bekenstein bound are the direct results of GSL. Therefore, we conclude that both of

${ }^{2}$ The weirdness of the phantom fluid, which seems to violate the second law of thermodynamics in many ways has been considered in [42-48] and may turn out to be completely un-physical. them must lead to the same entropy bound when imposing on a certain matter system. The cosmological constant is known to contribute to the metric as the $r^{2}$ term and for this contribution the D-bound is identified with the Bekenstein bound in dilute systems, in complete agreement with the above-mentioned conclusion. Any deviation from the $r^{2}$ term corresponding to the contributions of exotic quintessence-like and phantom-like fields such as $r$ and $r^{3}$ terms leads to D-bounds looser and tighter than the Bekenstein bound, respectively. Therefore, the quintessence-like and phantom-like fields are ruled out again because of the non-agreement of the D-bound and the Bekenstein bound. These features turn out to be a consequence of weakness and strongness of repulsion forces corresponding to quintessence-like and phantom-like fields in comparison to the repulsion force corresponding to the cosmological constant.

\section{REFERENCES}

[1] Weinberg S., Rev. Mod. Phys., 61 (1989) 1.

[2] Steinhardt P. J., in Critical Problems in Physics, edited by Fitch V. L. and Marlow R. (Princeton University Press, Princeton, NJ) 1997; Zlatev I., Wang L. M. and Steinhardt P. J., Phys. Rev. Lett., 82 (1999) 896; Steinhardt P. J., Wang L. M. and Zlatev I., Phys. Rev. D, 59 (1999) 123504.

[3] Caldwell R. R., Dave R. and Steinhardt P. J., Phys. Rev. Lett., 80 (1998) 1582.

[4] Fujil Y., Phys. Rev. D, 26 (1982) 2580.

[5] Ford L. H., Phys. Rev. D, 35 (1987) 2339.

[6] Wetterich C., Nucl. Phys. B, 302 (1988) 668.

[7] Ratra B. and Peebles P. J. E., Phys. Rev. D, 37 (1988) 3406.

[8] Carroll S. M., Hoffman M. and Trodden M., Phys. Rev. D, 68 (2003) 023509.

[9] Singh P., Sami M. and Dadhich N., Phys. Rev. D, 68 (2003) 023522.

[10] Sami M. and Toporensky A., Mod. Phys. Lett. A, 19 (2004) 1509.

[11] Corda C., J. Mod. Phys. D, 18 (2009) 2275.

[12] Capozziello S. and Francaviglia M., Gen. Relativ. Gravit., 40 (2008) 357.

[13] Sotiriou T. P. and Faraoni V., Class. Quantum Grav., 25 (2008) 205002.

[14] Inagaky T., Nojiri S. and Odintsov S. D., JCAP, 06 (2005) 010.

[15] Sakharov A. D., Sov. Phys. Dokl., 12 (1968) 1040; Dokl. Akad. Nauk Ser. Fiz., 177 (1967) 70; Sov. Phys. Usp., 34 (1991) 394; Gen. Relativ. Gravit., 32 (2000) 365.

[16] Visser M., Mod. Phys. Lett. A, 17 (2002) 977.

[17] Padmanabhan T., Rep. Prog. Phys., 73 (2010) 046901.

[18] Jacobson T., Phys. Rev. Lett., 75 (1995) 1260.

[19] Padmanabhan T., A Physical Interpretation of Gravitational Field Equations, AIP Conference Proceedings, Vol. 1241 (AIP) 2010, p. 93; Entropy density of spacetime and thermodynamic interpretation of field equations of gravity in any diffeomorphism invariant theory, arXiv:hep-th/0903.1254.

[20] Padmanabhan T., Mod. Phys. Lett. A, 25 (2010) 1129. 
[21] Padmanabhan T., Class. Quantum Grav., 21 (2004) 4485.

[22] Padmanabhan T., Emergence and Expansion of Cosmic Space as due to the Quest for Holographic Equipartition, arXiv:hep-th/1206.4916v1.

[23] Bekenstein J. D., Phys. Rev. D, 23 (1981) 287.

[24] Fischler W. and Susskind L., Holography and Cosmology, arXiv:hep-th/9806039.

[25] Easther R. and Lowe D. A., Phys. Rev. Lett., 82 (1999) 4967.

[26] Veneziano G., Phys. Lett. B, 454 (1999) 22.

[27] BAK D. and REy S. J., Class. Quantum Grav., 17 (2000) L83.

[28] Kaloper N. and Linde A., Phys. Rev. D, 60 (1999) 103509.

[29] Brustein R., Phys. Rev. Lett., 84 (2000) 2072.

[30] 'T Hooft G., Dimensional reduction in quantum gravity, arXiv:gr-qc/9310026.

[31] Susskind L., J. Math. Phys., 36 (1995) 6377.

[32] Corley S. and Jacobson T., Phys. Rev. D, 53 (1996) 6720 .

[33] Bousso R., JHEP, 01 (2001) 04.

[34] Bousso R., JHEP, 07 (1999) 004.

[35] Misner C. W. and Sharp D. H., Phys. Rev., 136B (1964) 571.

[36] Komar A., Phys. Rev., 113 (1959) 934; 129 (1963) 1873.

[37] Kiselev V. V., Class. Quantum Grav., 20 (2003) 1187.

[38] Majeed B., Jamil M. and Pradhan P., Adv. High Energy Physics, 2015 (2015) 124910; Shahzad M. U. and JAWAd A., Eur. Phys. J. C, $7 \mathbf{7}$ (2017) 372; Heydarzade Y. and Darabi F., Phys. Lett. B, 771 (2017) 365.
[39] Hellerman S. S., Kaloper N. and Susskind L., JHEP, 06 (2001) 003; Zlatev I., Wang L. and Steinhardt P. J., Phys. Rev. Lett., 82 (1999) 5; Vikman A., Phys. Rev. D., 71 (2005) 023515; Urena-López, L. A. and Matos T., Phys. Rev. D, 62 (2000) 081302; Sahni V., Lect. Notes. Phys., Vol. 653 (Springer) 2004, p. 141; Caldwell R. R., Dave R. and Steinhardt P. J., Phys. Rev. Lett., 80 (1998) 1586.

[40] Vikman A., Phys. Rev. D., 71 (2005) 023515; Singh P., Sami M. and Dadhich N., Phys. Rev. D, 68 (2003) 023522; Caldwell R. R., Phys. Lett. B, 545 (2002) 23; Carroll S. M., Hoffman M. and Trodden M., Phys. Rev. D, 68 (2003) 023509.

[41] Caldwell R. R., Phys. Lett. B, 545 (2002) 23; CaldWell R. R., Kamionkowski M. and Weinberg N. N., Phys. Rev. Lett., 91 (2003) 071301; NoJiri S., Odintsov S. D. and Tsujikawa S., Phys. Rev. D, 71 (2005) 063004 .

[42] Brevik I., Nojiri S., Odintsov S. D. and Vanzo L., Phys. Rev. D, 70 (2004) 043520.

[43] Gonzalez-Diaz P. F. and Siguenza C. L., Nucl. Phys. B, 697 (2004) 363.

[44] Hsu D. H., Jenskins A. and Wise M. B., Phys. Lett. B, 597 (2004) 270.

[45] Lima J. A. S. and Alcaniz J. S., Phys. Lett. B, 600 (2004) 191.

[46] Mosheni Sadjadi H., Phys. Rev. D, 73 (2006) 0635325.

[47] Nojiri S. and Odintsov S. D., Phys. Rev. D, 70 (2004) 103522.

[48] Nojiri S. and Odintsov S. D., Phys. Lett. B, 595 (2004) 1. 